\title{
Los condicionamientos pragmáticos del uso de los deícticos de tiempo en español
}

\author{
The pragmatic conditioning of the use \\ of time deictics in Spanish
}

\author{
Beata Brzozowska-Zburzyńska \\ Uniwersytet Marii Curie-Skłodowskiej w Lublinie (Polonia) \\ beata.brzozowska-zburzynska@poczta.umcs.lublin.pl
}

\begin{abstract}
The objective of this study is to reflect on the Spanish time expressions that function as deictic elements. Deixis is a phenomenon strictly related to aspects of a pragmatic nature since it depends on the situational context in which the participants of the communicative act are. We want to search, discover and describe the pragmatic conditioning that determines the appearance and operation of this type of element in Spanish. Our idea is to demonstrate that in time deictic expressions the semantic content is not enough for speakers to use these expressions properly. It is necessary to resort to information that exceeds the level of meaning and find guidelines for the good interpretation of the expression in the situational and personal context. The group of time deictics is not heterogeneous from the formal point of view. It includes simple elements (for example, adverbs) and more or less complex elements (noun and prepositional phrases). We will focus on this second group and, above all, on the nominal constructions that include determinants and temporal adjectives since in this type of structure each constituent element performs a different semantic-pragmatic function and only the combination of these allows us to discover the definitive value of the set.
\end{abstract}

Keywords: deictics, time, pragmatics

\section{INTRODUCCIÓN}

Principalmente se reconoce que los deícticos son adverbios y pronombres de tiempo y lugar que permiten situar las acciones verbales en referencia a las coorde- 
nadas espacio-temporales. Sin embargo, en este estudio nos dedicaremos a presentar expresiones lingüísticas que pueden funcionar como deícticas pero cuya estructura formal es más o menos compleja. Serán, por lo tanto, casos de elementos menos prototípicos de la categoría funcional de los deícticos, y, más concretamente, las expresiones temporales de base nominal. Por eso, en este artículo nos gustaría explorar con más detalles la deíxis de tiempo y buscar los condicionamientos pragmáticos que influyen en la adecuada interpretación de estas expresiones. Como demostraremos a continuación, su contenido semántico no es suficiente para que los hablantes las identifiquen correctamente.

\section{LA DEÍXIS Y LOS DEÍCTICOS TEMPORALES}

La deíxis empezó a interesar a los lingüistas, con más énfasis, en los años 30 del siglo XX, es decir, a partir de los trabajos de Karl Bühler y su Teoría del lenguaje (1934), donde el lingüista alemán expuso la definición y la primera clasificación de los fenómenos relacionados con esta función enunciativa del lenguaje. Lo siguieron otros investigadores (Benveniste, 1966; Carbonero Cano, 1979; Lyons, 1980; Tanz, 1980 entre muchos otros), quienes han tratado el tema de la existencia de elementos lingüísticos cuya función principal es mostrativa y no simbólica, y cuya interpretación no depende, solamente, de las características semánticas sino, sobre todo, de su vinculación con el contexto, entendido de una manera muy amplia ${ }^{1}$. La deíxis es, por lo tanto, un fenómeno pragmático, ya que permite codificar la relación entre la lengua y el contexto del uso (Reyes, Baena, Urios, 2005, p. 121) o, dicho de otro modo, “colocar en el espacio y en el tiempo una sustancia semántica" (Marcos Marín, 1980, citado en Cifuentes, 1989, p. 71).

Sin embargo, según los investigadores que siguen las pautas de la lingüística cognitiva, se reconoce el estatus tanto semántico como pragmático de cualquier entidad lingüística, por lo que la separación de ambas disciplinas resulta, a veces, difícil, o incluso, imposible. Aquí seguiremos la postura que adoptan muchos de los lingüistas cognitivistas, que se resume del modo siguiente: "una perspectiva semántica, de base pragmática, será necesaria para el estudio de cualquier entidad lingüística" (Cifuentes Honrubia, 1994, p. 138). Así también entendemos el fenómeno de la deixis, que se puede articular a través de las expresiones lingüísticas pertenecientes a tres categorías: determinantes, relacionantes y verbos. Las expresiones que nos ocupan en este artículo pertenecen al segundo grupo, es decir, a la clase semántica de los relacionantes temporales -análoga a la de los relacionantes espaciales, que Cifuentes Honrubia (1989) llamó locativos espaciales-.

${ }^{1}$ Paveau \& Sarfati (2009, p. 245) aseguran que en el marco de la pragmática se estudian hasta cuatro tipos de contextos diferentes: el situacional, el cultural, el interactivo y el epistémico. 
Podemos admitir que los deícticos son ese tipo de expresiones que, guardando su significado semántico, designan diferentes referentes según la situación discursiva en la que aparecen. En realidad, la identificación del referente no es posible fuera del contexto. Si alguien recibe una nota en la que se dice "vuelvo mañana", pero desconoce el momento aproximativo en el cual el remitente la preparó, no es capaz de interpretar adecuadamente lo escrito, ya que mañana es un adverbio de tiempo deíctico que indica el día siguiente al del acto comunicativo y su interpretación es posible solamente si conocemos las coordenadas temporales del acto de habla.

Los deícticos de tiempo en español son todo tipo de expresiones que permiten situar las acciones verbales a partir del momento del habla o en referencia a este momento del habla. Sin embargo, no todos tienen el mismo estatus y funcionamiento:

Los deícticos temporales pueden tener un punto de referencia objetivo y delimitado, sometido a medida cronológica, incluyendo hoy en oposición a ayer o mañana, por ejemplo. Otro grupo de adverbios deícticos de tiempo no tiene una medición precisa. A este grupo pertenece ahora, que puede incorporar el momento de la palabra, pero tiene límites vagos, y se opone a antes, luego, después, y entonces como las situaciones que no corresponden al ahora (Silva-Corvalán, 2001, p. 220).

Los casos prototípicos de esta clase de elementos lingüísticos serían, como acabamos de ver, los adverbios. Sin embargo, como reconoce Carbonero Cano (1979, p. 115):

(...) por evidentes razones de economía del lenguaje, el sistema temporal no dispone de una unidad adverbial mínima de tipo léxico, para cada situación temporal que haya que expresar. (...) Esa es la razón por la que encontramos que en el cuadro general del sistema adverbial hay algunas «casillas vacías» cuya noción no dispone de unidad específica y habrá de ser llenada por otra unidad más compleja que la exprese.

Este sería, por lo tanto, el papel que desempeñan las expresiones temporales, entre ellas, las de base nominal, es decir, el de llenar las casillas vacías y marcar las relaciones temporales más complejas y más específicas.

\section{ESTRUCTURAS DEÍCTICAS EN ESPAÑOL}

Las expresiones temporales de base nominal son sintagmas nominales o preposicionales más o menos complejos, en los cuales el sustantivo desempeña el papel de núcleo y es obligatorio. Otros elementos constitutivos pueden aparecer de modo facultativo.

Cuando empleamos estas expresiones efectuamos, necesariamente, varias operaciones mentales que nos llevan a la interpretación adecuada de la expresión. Cada 
elemento lingüístico que forma parte de una expresión tal aporta otro tipo de información semántica que debe ser actualizada contextualmente. Así, los sustantivos dan cuenta de la división convencional de las entidades temporales en periodos más o menos determinados por una comunidad socio-lingüístico-cultural. Las preposiciones configuran conceptualmente las entidades temporales como contenedores, estructuras complejas o duraciones. Los determinantes y otros elementos adyacentes establecen la conexión entre la entidad temporal y su fondo de anclaje, el cual corresponde al acto mismo de la comunicación y todos sus componentes. En la nomenclatura empleada por Langacker (2009) son predicaciones de anclaje (p. 337).

Todas estas expresiones temporales de base nominal pueden desempeñar la función de los elementos deícticos, pero también pueden indicar una relación no deíctica, cuando el punto de referencia no se encuentra en la situación discursiva sino que aparece determinado contextualmente o cotextualmente fuera del acto comunicativo. Lo que permite distinguir ambos tipos de funcionamiento son, a veces, algunos elementos gramaticales, $\mathrm{y}$, otras veces, informaciones de tipo pragmático.

Desde el punto de vista semántico, las expresiones de tiempo pueden desempeñar varias funciones. Haspelmath (1997), quien estudió los adverbios de tiempo en más de 50 lenguas del mundo, propuso las siguientes funciones para el análisis de estas expresiones:

- localización simultánea,

- localización secuencial (anterior, posterior),

- localización secuencial durativa (anterior, posterior),

- distancia temporal (anterior, posterior),

- extensión temporal (atélica, télica).

Entre ellas encontramos las que son deícticas como, por ejemplo, esta mañana, las que son anafóricas, por ejemplo, al día siguiente, y las que podríamos llamar flexibles porque según el contexto pueden funcionar como deícticas o no deícticas, por ejemplo: el jueves (García Fernández, 2013, pp. 56-57). Desde el punto de vista formal, en español podemos hablar de tres estructuras fundamentales: determinante + sustantivo, determinante $+\mathrm{SN}$, preposición $+\mathrm{SN}$. A continuación, presentaremos algunas particularidades de las expresiones temporales españolas, según su estructura.

En primer lugar, vamos a analizar las expresiones que contienen el artículo y el sustantivo solo. En español, este tipo de construcciones son poco frecuentes y aparecen, principalmente, en el caso de los sustantivos que designan los días de la semana ${ }^{2}$. Estos últimos no pueden aparecer solos (como, por ejemplo, en francés) porque, como asegura Hortensia Martínez (1996, p. 33), no son autónomos semánticamente, por lo que requieren que se efectúe un tipo de anclaje especial. En este caso, es el artículo lo que garantiza la vinculación del sustantivo con el fondo de anclaje. Como explica

\footnotetext{
${ }^{2}$ Es posible que aparezca esta construcción en el caso de los sustantivos víspera y antevíspera, pero entonces no es posible su interpretación deíctica por el contenido semántico de éstos.
} 
Martínez Vázquez (2006, p. 415), "el valor del artículo se define con referencia a tres principios clave: contacto mental, unicidad y maximalidad". Este último está relacionado con el empleo de los sustantivos en plural. Hablamos del contacto mental cuando el hablante considera que el oyente, gracias a las indicaciones de tipo pragmático, es capaz de constatar que el referente está presente en el contexto al que se refiere la expresión nominal. El principio de unicidad, a su vez, implica que el referente es el único accesible en el contexto en el cual se desarrolla el acto comunicativo.

Cuando el nombre de un día de la semana aparece con el artículo definido, entonces la expresión así formada desempeña la función de simultaneidad. El artículo permite la identificación del día del que se habla o bien en referencia al momento del habla y entonces hablamos de su empleo deíctico, o bien, en referencia a otro momento evocado por el contexto. Podríamos representar el esquema de este conjunto del modo siguiente (Brzozowska-Zburzyńska, 2017, p. 178):

ARTÍCULO = nombre del día de la semana en contexto (deíctico o anafórico).

NOMBRE $=$ nombre correspondiente a un día que forma parte del conjunto de días constitutivos de la unidad superior que es semana.

ARTÍCULO + NOMBRE = un día de la semana concreto más próximo al momento del habla (empleo deíctico) o a un momento indicado por el contexto (empleo anafórico).

(1) Nos veremos el lunes.

(2) Llegan el fin de semana del 2 de julio. El lunes ya estarán con nosotros en la playa.

En los ejemplos (1) y (2) empleamos el mismo sintagma bimembre el lunes. En el primer caso, con esta expresión nos referimos al lunes más próximo al momento del habla, por eso, no hace falta especificar con más detalles las coordenadas temporales. No es así en el segundo ejemplo, donde la identificación del día se hace a través de la interpretación de otra expresión temporal antecedente (el fin de semana del 2 de julio), cuya identificación requiere que los hablantes compartan los mismos conocimientos sobre su ubicación temporal.

Otros sustantivos temporales, como semana, mes, año, día pueden formar construcciones con el artículo, sin preposición, pero a condición de que aparezca algún otro elemento modificador (adjetivo, complemento del nombre). Es así porque la identificación de estas entidades con su dominio directo no es uni-unívoca (Wilk-Racięska, 2009, p. 107) como en el caso de un día de la semana ${ }^{4}$. Una semana, un mes, un año, o un día se incluyen en el marco de las entidades temporales superiores, pero

\footnotetext{
${ }^{3}$ El esquema ha sido elaborado a partir del propuesto por Martínez Vázquez (2006, p. 216), que representa el proceso de fusión de los conceptos semántico simples que se efectúa en el caso de una entidad bimembre.

${ }^{4}$ El lunes pertenece al dominio de la semana y como en una semana puede haber solamente un lunes, la identificación se hace de un modo directo e inequívoco (Brzozowska-Zburzyńska, 2017, p. 178).
} 
no son elementos únicos (en una semana hay siete días, en un año - doce meses, etc.), por lo que se establecen relaciones multi-unívocas y es necesario que se especifiquen estas con la ayuda de otros elementos lingüísticos, como los demostrativos o expresiones anafóricas complejas (aquella semana, la semana que viene, el próximo año).

Como acabamos de observar, las expresiones temporales de base nominal también se pueden formar con el adjetivo demostrativo. En tal caso ya no hay restricciones de coaparición con todos los sustantivos temporales y en varias situaciones el demostrativo resulta ser un elemento indispensable para que la expresión temporal pueda desempeñar la función semántica de simultaneidad.

Tanto el artículo como el demostrativo contienen la noción de la definitud que añaden al significado del sustantivo al que acompañan. Pero el demostrativo añade el matiz semántico de localización, por lo que resulta más apropiado o, incluso, imprescindible en los contextos que requieren más precisión espacial o temporal. Los demostrativos marcan, por lo tanto, el grado de la identificación del objeto concebido, así que se deben entender como elementos obligatoriamente deícticos (Data-Bukowska, 2005, pp. 65-66).

Como ya hemos dicho antes, los demostrativos, al igual que los artículos, son elementos que participan activamente en lo que Langacker $(2009$, p. 85$)$ llama construcción de las escenas, que se efectúa teniendo en cuenta cuatro fenómenos importantes: concreción/especificación, focalización, prominencia y perspectiva. Gracias a ellos, el hablante acentúa diferentes aspectos de la situación que quiere describir teniendo en cuenta sus preferencias subjetivas e individuales y también condiciones y propósitos de la comunicación (Data-Bukowska, 2005, p. 248).

El paradigma de los demostrativos españoles es tripartito tanto en el dominio espacial como en el temporal, pues cada elemento de este paradigma corresponde a diferentes modos de conceptualización de la situación. Creemos que para entender bien el funcionamiento de este sistema complejo de los demostrativos españoles puede ser válida la propuesta de Delbecque (2013, p. 108), quien propuso la distinción de tres posibles perfiles que nosotros adoptamos para el estudio de la dimensión temporal:

(i) una simple captación contingente, sin a priori, con una base de conocimiento mínimamente activada (est-),

(ii) la fijación de una imagen anclada en la base de conocimientos personales (es-),

(iii) la aceptación de un contorno global, con base objetivada (aquel-).

Vamos a analizar a continuación tres oraciones en las cuales aparecen sintagmas nominales de tiempo con valor deíctico que contienen un demostrativo:

(3) Un incendio forestal activo iniciado esta madrugada en Arbo (Pontevedra) ha calcinado de momento unas 90 hectáreas 5 .

\footnotetext{
${ }^{5} \mathrm{http}: / /$ www.laregion.es/articulo/galicia/incendio-activo-iniciado-madrugada-ha-quemado-90-hectareas-arbo/20160802123510639086.html, consulta el 2 de agosto de 2016.
} 
(4) Los niños salen del colegio y al llegar a casa encuentran en sus ordenadores un email con los problemas que deben resolver esa tarde $\left(\mathrm{CREA}^{6}\right)$.

(5) Aquella mañana vi cómo la gente se volcaba, tiraba cosas por las ventanas. (...) Como esa chica rumana con la que siempre bajaba en Nuevos Ministerios y que ese día le cuidó hasta que le evacuaron al hospital Clínico (CREA).

En el ejemplo (3) aparece el sintagma nominal esta madrugada, que no requiere la activación de ninguna información adicional si la recibimos el día de la publicación de la noticia en la que apareció. Esta entidad temporal acompañada del demostrativo esta significa que 'la madrugada pertenece al mismo día que el de la enunciación de la expresión'. Por eso, su identificación se hace con referencia al momento del habla y su contigüidad temporal inmediata. En este caso concreto se trata de una noticia periodística publicada en una página web dedicada a emitir noticias de Galicia fechada en el mes de agosto del año 2016. Si la leemos hoy, ya es necesario buscar este punto de referencia temporal para no interpretar erróneamente lo escrito.

De modo general debemos observar que la identificación de las entidades temporales que se emplean con el demostrativo este siempre activa el dominio de una entidad superior a la que pertenece el intervalo identificado (Brzozowska-Zburzyńska, 2007, p. 189). Esto se refiere tanto a la expresión esta tarde como este lunes o este mes. Para la tarde, la entidad superior sería el día; para el lunes, la semana; para el mes, el año. Sin embargo, en todos estos casos la interpretación no resulta inequívoca. Como las entidades complejas se componen de varios elementos que aparecen en un orden bien determinado, pueden darse equivocaciones. Así, en el momento de decir este verano, tanto me puedo referir al verano que ya pasó como al que viene. Por lo tanto, para ser entendido, debo asegurarme de que el interlocutor comparta conmigo las mismas informaciones previas y que no se equivoque en la interpretación de mi acto comunicativo.

En el ejemplo (4) aparece la expresión esa tarde. Su identificación no es inmediata, porque este sintagma no se refiere al momento del habla. El remitente de esta oración puede hablar, en este caso, de un suceso único, pero también puede referirse a acciones que se suelen repetir, como si fuera una instrucción más general. Por lo tanto, la búsqueda del punto de referencia a partir del cual se puede efectuar la identificación de la expresión esa tarde se hace a través de otros indicadores temporales incluidos en el discurso. Aparece la contigüidad inmediata con el momento de referencia y la expresión esa tarde significa en esta oración 'la misma tarde que la de salir del colegio y llegar a casa'.

En el ejemplo (5), la expresión aquella mañana designa una entidad temporal que no es identificable de modo objetivo y es exterior al espacio temporal más próximo a los participantes del acto comunicativo. Además, en este ejemplo aparecen dos

\footnotetext{
${ }^{6}$ CREA, Corpus de Referencia del Español Actual.
} 
expresiones con dos demostrativos: aquella mañana y ese día. Ambas expresiones se refieren, objetivamente, al mismo día, pero su interpretación es diferente. Con la expresión aquella mañana, el hablante quiere subrayar la lejanía temporal entre el momento de habla y la situación descrita. Creemos también que en esta expresión se incluyen algunos sentimientos relacionados con lo experimentado en el pasado ${ }^{7}$. La expresión ese día a su vez, se identifica en referencia al momento indicado por la expresión aquella mañana, por lo que tenemos aquí un caso especial de anáfora asociativa.

Hemos dicho que las construcciones con el demostrativo son posibles, en español, con la mayoría de los sustantivos temporales. Pueden aparecer tanto en las construcciones sin preposición como en las prepositivas. A veces, compiten con ellas.

(6) Nos vimos varias veces esta semana/durante esta semana.

La preposición es obligatoria si el demostrativo acompaña a los sustantivos que designan entidades temporales imprecisas o que son menores a una hora, porque de esta forma se indica la discontigüidad del intervalo: en ese momento, en aquel instante, a esa hora, en aquel minuto, en este mismo segundo, etc. El grado de accesibilidad de estas entidades temporales no es estable y, muchas veces, depende de las coordenadas de tipo pragmático. Por eso, las construcciones que solamente contienen la preposición en y el sustantivo no son frecuentes, ya que su grado de accesibilidad no es suficiente y es imprescindible que aparezca un elemento adyacente. Una excepción la constituyen los nombres de los meses o de las estaciones del año que pueden construir una expresión con preposición, pero sin determinante (Brzozowska-Zburzyńska, 2007, pp. 212-213):

(7) Me lo comunicaron en junio.

(8) En junio nos vamos de vacaciones.

(9) Volverán en marzo.

(10) En verano solemos viajar.

(11) En invierno nos gusta esquiar.

Los ejemplos que acabamos de enumerar pueden ser interpretados de dos maneras diferentes: de modo deíctico y de modo reiterativo. En estos casos, para interpretar adecuadamente los referentes temporales de las expresiones es necesario recurrir al contexto del habla y también al conocimiento general del mundo, es decir, a informaciones que sobrepasan el nivel exclusivamente semántico.

\footnotetext{
${ }^{7}$ Esta oración se relaciona estrechamente con el atentado del 11 de marzo en Madrid en 2004.
} 


\section{CONCLUSIONES}

En el estudio que acabamos de presentar hemos querido subrayar el valor tanto semántico como pragmático de las expresiones de tiempo que pueden desempeñar la función de elementos deícticos. Creemos haber conseguido este fin comprobando que la adecuada interpretación de este tipo de expresiones depende tanto de su conceptualización semántica como de la información que, gracias a los elementos gramaticales empleados, los hablantes son capaces de sacar de las coordenadas situacionales y extralingüísticas que influyen en el acto comunicativo. No hemos podido profundizar el estudio de las expresiones preposicionales de tiempo, pero queremos subrayar que algunas de ellas también pueden desempeñar el papel de elemento deíctico, no solamente en la función semántica de la simultaneidad, sino también en otras funciones donde el uso de una preposición concreta ya garantiza la lectura deíctica o no deíctica de la expresión. Este es, por ejemplo, el caso de la función de la distancia posterior donde la expresión dentro de un día es deíctica mientras que la de un día después/más tarde no lo es. Por eso, tenemos la intención de seguir investigando el funcionamiento deíctico de las expresiones temporales en nuestras futuras publicaciones. 


\section{BIBLIOGRAFÍA}

Benveniste, E. (1966). Problèmes de linguistique générale, 1. Paris: Gallimard.

Brzozowska-Zburzyńska, B. (2017). Estudio contrastivo de las expresiones temporales de base nominal en español, francés y polaco. Lublin: Wydawnictwo UMCS.

Bühler, K. (1934). Sprachtheorie. Die Darstellungsfunktion der Sprache. Jena: Fischer; traducido por J. Marías: Teoría del lenguaje, Madrid: Revista de Occidente, 1950.

Carbonero Cano, P. (1979). Deíxis espacial y temporal en el sistema lingüístico. Sevilla: Universidad de Sevilla.

Cifuentes Honrubia, J.L. (1989). Lengua y espacio. Introducción al problema de la deixis en español. Alicante: Universidad de Alicante.

Cifuentes Honrubia, J.L. (1994). Gramática cognitiva. Fundamentos críticos. Madrid: Eudema.

Data-Bukowska, E. (2005). Zaimki wskazujące w języku szwedzkim. Analiza znaczeń z perspektywy językoznawstwa kognitywnego. Kraków: Oficyna Wydawnicza TEXT.

Delbecque, N. (2013). Anclaje experiencial y epistémico de los demostrativos no situacionales en español. Anuario de Letras, 1, 85-170. DOI: 10.1016/S0185-1373(13)70255-6.

García Fernández, L. (2013). El tiempo en la gramática. Madrid: Arco/Libros.

Haspelmath, M. (1997). From Space to Time. Temporal Adverbials in the World's Languages. MünchenNewcastlle: Lincom Europa.

Langacker, R. (2009). Gramatyka kognitywna. Wprowadzenie. Kraków: Universitas.

Lyons, J. (1980). Semántica, Barcelona: Teide.

Martínez García, H. (1996). Construcciones temporales. Madrid: Arco Libros.

Martínez Vázquez, R. (2006). Elementos de gramática cognitiva en la sintaxis del artículo de Apolonio Díscolo. HABIS, 37, 413-425. DOI: 10.12795/Habis.2006.i37.29.

Paveau, M.-A., Sarfati, G.-É. (2009). Wielkie teorie językoznawcze. Od językoznawstwa historyczno-porównawczego do pragmatyki. Kraków: Flair-Avalon.

Reyes, G., Baena, E., Urios, E. (2005). Ejercicios de pragmática II. Madrid: Arco/Libros.

Silva-Corvalán, C. (2001). Sociolingüistica y pragmática del español. Washington: Georgetown University Press.

Tanz, Ch. (1980). Studies in the acquisition of deictic terms. Cambridge: Cambridge University Press.

Wilk-Racięska, J. (2009). Od wizji świata do opisu językoznawczego w kategoriach lingwistyki kulturowej. Uwagi na temat hiszpańskiej syntagmy nominalnej. Katowice: Wydawnictwo Uniwersytetu Śląskiego. 